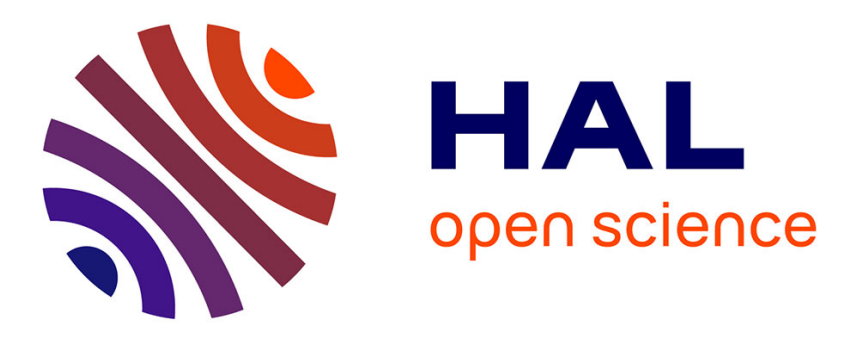

\title{
Rapid characterization of neutral polymer brush with a conventional zetameter and a variable pinch of salt
}

Alexandre Morin, Antoine Aubret, Mena Youssef, Stefano Sacanna, Jérémie Palacci

\section{- To cite this version:}

Alexandre Morin, Antoine Aubret, Mena Youssef, Stefano Sacanna, Jérémie Palacci. Rapid characterization of neutral polymer brush with a conventional zetameter and a variable pinch of salt. Soft Matter, 2020, 16 (17), pp.4274-4282. 10.1039/C9SM01850F . hal-03279171

\section{HAL Id: hal-03279171 \\ https://hal.science/hal-03279171}

Submitted on 6 Jul 2021

HAL is a multi-disciplinary open access archive for the deposit and dissemination of scientific research documents, whether they are published or not. The documents may come from teaching and research institutions in France or abroad, or from public or private research centers.
L'archive ouverte pluridisciplinaire HAL, est destinée au dépôt et à la diffusion de documents scientifiques de niveau recherche, publiés ou non, émanant des établissements d'enseignement et de recherche français ou étrangers, des laboratoires publics ou privés. 


\title{
Soft Matter
}

\section{ARTICLE TYPE}

Cite this: DOI: $10.1039 / x x x x x x x x x x$

\section{Rapid characterization of neutral polymer brush with a conventional zetameter and a variable pinch of salt}

\author{
M. Youssef, ${ }^{a \ddagger}$ A. Morin, ${ }^{* a b \ddagger}$ A. Aubret,${ }^{b}$ S. Sacanna ${ }^{a}$ and J. Palacci ${ }^{* b}$
}

Received Date

Accepted Date

DOI: $10.1039 / x x x x x x x x x x$

www.rsc.org/journalname

\begin{abstract}
The fundamental and practical importance of particle stabilization has motivated various characterization methods for studying polymer brushes on particle surfaces. In this work, we show how one can perform sensitive measurements of polymer coating on colloidal particles using a commercial zetameter and salt solutions. By systematically varying the Debye length, we study the mobility of the polymer-coated particles in an applied electric field and show that the electrophoretic mobility of polymer-coated particles normalized by the mobility of non-coated particles is entirely controlled by the the polymer brush and independent of the native surface charge, here controlled with $\mathrm{pH}$, or the surface-ion interaction. Our result is rationalized with a simple hydrodynamic model, allowing for the estimation of characteristics of the polymer coating: the brush length $L$, and the Brinkman length $\xi$, determined by its resistance to flows. We demonstrate that the Debye layer provides a convenient and faithful probe to the characterization of polymer coatings on particles. Because the method simply relies on a conventional zetameter, it is widely accessible and offers a practical tool to rapidly probe neutral polymer brushes, an asset in the development and utilization of polymer-coated colloidal particles.
\end{abstract}

\section{Introduction}

The stabilization of colloidal suspensions, achieved by an adequate balance of attractive van der Waals interactions with repulsive forces ${ }^{1}$, is an essential aspect of colloidal science. Stable particles can be used as building blocks for macroscopic materials with properties that are engineered from the interactions between micrometric constituents. Self-assembly by tailored shapes ${ }^{2-4}$ or specific interactions ${ }^{5-8}$ has shown promise for man-made materials with unique optical ${ }^{9}$ or photonic ${ }^{10}$ properties.

Steric repulsion is a common mechanism that prevents the aggregation of particles in suspension and can be achieved by coating particles with a near-impenetrable polymer brush. The characterization of polymer brushes has been the focus of theoretical investigations ${ }^{11-13}$ and experimental characterization using adsorption isotherm, optics (ellipsometry or photon correlation spectroscopy), rheology, NMR solvent proton relaxation, and smallangle neutron scattering, see for example the recent review ${ }^{14}$ and references therein. Probing the properties of nanoscopic brush is challenging and often requires cumbersome equipment. For example, ellipsometry uses the change of polarization of light upon reflection or transmission to measure dielectric properties of sur-

\footnotetext{
${ }^{a}$ Molecular Design Institute, Department of Chemistry, New York University, New York, NY 10003, USA. E-mail: alexandre.morin@nyu.edu

${ }^{b}$ Department of Physics, University of California, San Diego, USA. E-mail: palacci@physics.ucsd.edu

$\$$ These authors contributed equally to this work
}

faces. Alternatively, photon correlation spectroscopy does not rely directly on optics. The adsorbed layer thickness is determined by comparing the diffusion coefficient of bare and polymer-coated particles, which can reveal finicky when nanoscale coatings are probed on micrometric beads ${ }^{15}$ rather than nanoparticles.

Phoretic interfacial phenomena naturally bridge macroscopic observable with the nanoscopic range of the interactions they arise from. They constitute straightforward probes to the nanoscale, sensitive to minute changes in surface properties ${ }^{16,17}$. For example, phoretic flows are significantly affected by hydrodynamic slip of a few nanometers ${ }^{18,19}$, and can be suppressed by nanometric surface roughness ${ }^{20}$. In electrophoresis, the nanometric scale is set by the Debye length, the distance at which the surface charge of the particle is screened by a double layer of ions. The electrical double layer is set in motion following the application of an external electric field $E_{0}$. The mechanical balance between this driving force and the viscous dissipation sets the amplitude of the electrophoretic velocity, $\mathbf{v}_{\mathrm{EP}}=\mu E_{0}$, where $\mu$ is the electrophoretic mobility. The presence of polymer brush affects the electrophoretic mobility of a particle by increasing the viscous drag near the particle surface ${ }^{21-27}$, providing a practical mean to alter the electrophoretic response of a system. For example, a thermo-responsive polymer can be used to stop the electrokinetic flow in microchannels by swelling of the polymer ${ }^{28,29}$. The particular relationship between the polymer brush and changes in electrophoretic mobility of particles 
has been investigated by others $24,25,27,28,30,31$, and discussed for neutral polymer and polyelectrolytes ${ }^{26,32}$. Electrophoretic measurements were used to study adsorption of block-copolymers on various colloidal particles, e.g. silica, latex, at different $\mathrm{pH}$, using a model that assumes infinite friction in the brush ${ }^{30}$. Additionally, studies using a hairy layer accounted for the finite permeability of polymer brushes on particles ${ }^{24}$ or liposomes ${ }^{25,33}$ by varying the salt concentration.
(A)

Low salt

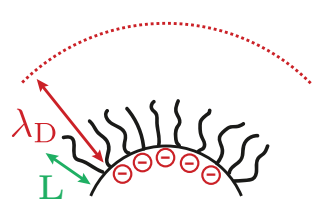

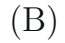

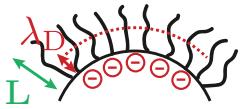

High salt
(C)

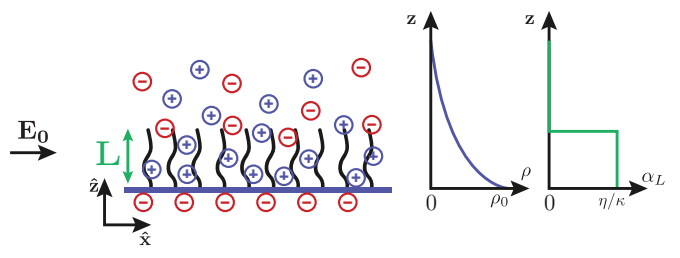

Fig. 1 Exploiting the Debye length to probe nanoscopic polymer brushes. (A) At low salt concentrations, the Debye length $\lambda_{D}$ typically extends much farther than the length $L$ of the polymer brush. (B) Increasing the salt concentration reduces the Debye length, which eventually becomes comparable to the length of the polymer brush. (C) As an electric field $\mathbf{E}_{\mathbf{0}}$ is applied, the motion of the double layer of charge density $\rho$ is influenced by the presence of the polymer. The change in electrophoretic mobility upon reduction of the Debye length carries information specific to the polymer brush: its length $L$ and its permeability $\kappa$. In a minimal model, the density of charge decays exponentially from the surface $\rho(z)=\rho_{0} \exp \left(-z / \lambda_{\mathrm{D}}\right)$ and the brush offers a homogeneous resistivity to the flow $\forall z \leq L \quad \alpha_{L}(z)=\eta / \kappa$ where $\eta$ is the solvent viscosity.

In this work, we vary the Debye layer and measure electrophoretic mobilities to probe neutral polymer-coatings. We show that the measured mobilities can be adequately normalized to provide a quantity that is uniquely set by characteristics of the polymer layer. We demonstrate that the normalized mobility does not depend on the native charge of the substrate particle, by changing the $\mathrm{pH}$ of the solution, nor on the interactions between the grafted colloids and the ions present in solution. Our approach allows for the determination of the hydrodynamic fingerprint of a brush, its thickness and permeability, defined by the properties of the polymer coating. We compare the results with independent measurements of polymer length by dynamic light scattering that confirm our measurements and we estimate the brush density from its permeability.

As the Debye layer is given by $\lambda_{D}=\left(4 \pi \lambda_{\mathrm{B}} \sum_{i} c_{i} z_{i}\right)^{-1 / 2}$, where $\lambda_{\mathrm{B}}$ is the Bjerrum length, $c_{i}$ the mean concentration of ions $i$ in the solution, and $z_{i}$ their valence ${ }^{17}$, it is typically 1 to $15 \mathrm{~nm}$ in water and can be tuned to ensure sensitive measurements of polymer brush. Our strategy is summarized in Fig. 1. Charged colloidal particles are coated with neutral polymers, forming a brush of extension $L$. At low salt concentrations [Fig. 1(A)], the Debye length $\lambda_{\mathrm{D}}$ extends further than the brush and the brush barely affects the electrophoretic mobility of the particle. As the salt concentration $c$ increases [Fig. 1(B)], the Debye length $\lambda_{D} \propto 1 / \sqrt{c}$ decreases. As it gets comparable to the extent of the brush, the colocation of the electric driving force and the hydrodynamic drag results in a sensible reduction of the electrophoretic mobility. In the limit of a brush with infinite friction, the shear-plane shifts by $L$ from the particle surface, resulting in vanishing electrophoretic mobilities $\mu=0$ for $\lambda_{\mathrm{D}} / L \rightarrow 0$. Benefits of our approach are that it relies on a conventional zetameter, an apparatus widespread in physicochemistry laboratories, and that the Debye layer is conveniently controlled by the salt concentration (or ionic strength) of the solution. By investigating the mobilities for Debye layers of various lengths, we show that the normalized electrophoretic mobilities of polymer-coated particles with the mobility of polymer-free particles depends uniquely on the polymer brush. This experimental observation is rationalized by a simple model that eventually enables the estimation of the permeability and length of the brush. This result demonstrates the relevance of our approach and sets the Debye length as a faithful probe to study and compare neutral polymer brushes on particle surfaces.

\section{Methods}

We devise a modular synthesis to install polymer brushes of different lengths onto monodispersed spherical particles. The resulting model system consists of polymeric 3-(trimethoxysilyl)propyl methacrylate (TPM) microspheres with a grafted layer of PEO-PPO-PEO pluronic triblock copolymers: a hydrophobic poly(propylene oxide) (PPO) central block and two hydrophilic poly(ethylene oxide) (PEO) ends. Pluronic are neutral polymers with the high hydrophilicity of PEO caused by hydrogen bonding with water molecules ${ }^{34,35}$.

More precisely, TPM microspheres are synthesized following an emulsion polymerization route reported in the literature ${ }^{36}$ and schematized in Fig. 2(A). Under basic condition $(\mathrm{pH} \sim 9)$, the silyl moieties of TPM hydrolyse and condensate leading to the homogeneous nucleation of crosslinked liquid droplets. Once the final size of the droplets $(\sim 2 \mu \mathrm{m})$ is reached, PEO-PPOPEO block copolymers are added to the emulsion $(\sim 0.05 \mathrm{wt} \%)$. The polymers adsorb on the emulsion droplets, with the PPO blocks acting as 'anchors' and the PEO blocks as 'buoys' ${ }^{11}$. We henceforth refer to the resulting coating layer as a polymer brush $^{37,38}$. In the present work, we prepare bare TPM particles, i.e. without polymer coating, and TPM particles grafted with polymers of increasing PEO lengths: Pluronic F68, F88, and F108. They constitute a series of tri-block copolymers $\mathrm{H}\left[\mathrm{OCH}_{2} \mathrm{CH}_{2}\right]_{\mathrm{n}}\left[\mathrm{OCH}\left(\mathrm{CH}_{3}\right) \mathrm{CH}_{2}\right]_{\mathrm{m}}\left[\mathrm{OCH}_{2} \mathrm{CH}_{2}\right]_{\mathrm{n}} \mathrm{OH}$ with increasing molecular weight, $8400 \mathrm{~g} / \mathrm{mol}, 11800 \mathrm{~g} / \mathrm{mol}$ and $14000 \mathrm{~g} / \mathrm{mol}$, respectively at fixed ratio $\mathrm{n} / \mathrm{m}=0.4$. The TPM particles are then solidified, thereby grafting the adsorbed polymers, by taking advantage of the carbon-carbon double bond of the methacrylate moities: the radical polymerization is done by adding the initator, 2,2'-Azobis(2- methylpropionitrile) (AIBN) and heating the solution to $80^{\circ} \mathrm{C}$ for $\sim 3$ hours [Fig. 2(A)]. The particles are washed by several cycles of centrifugation and resuspension in water. Figure 2(B) shows the resulting TPM microspheres imaged by scanning electron miscroscopy. The particles are highly monodisperse with a diameter of $1.83 \pm 0.036 \mu \mathrm{m}$. Other batches of monodis- 
perse particles with $\sim 1 \mu \mathrm{m}$-diameter were also synthesized and used. Similarly to silica colloids, TPM colloids bear negative surface charges due to surface $\mathrm{Si}^{-} \mathrm{O}^{-}$groups, increasing with basic $\mathrm{pH}[$ Fig. 2(C)].

To characterize the polymer brushes from the electrophoretic response of the colloids, we use a commercial zetameter (Malvern Zetasizer NanoS) to perform electrophoretic measurements. In this work, we report electrophoretic mobility, the quantity directly measured by the zetameter, rather that the $\zeta$-potential, which relationship to the electrophoretic mobility is derived from a model. It is worth recalling that, in the case of particles much larger that their Debye layer and in the Gouy-Chapman model, the relationship is given by the Smoluchowski formula, and is simply $\zeta=\frac{\eta}{\varepsilon} \mu$, where $\varepsilon$ is the dielectric constant of water and $\eta$ its viscosity. In the remainder of this Article, we use electrophoretic measurements, which constitute our direct measure and do not depend on an inversion model used by the zetameter.

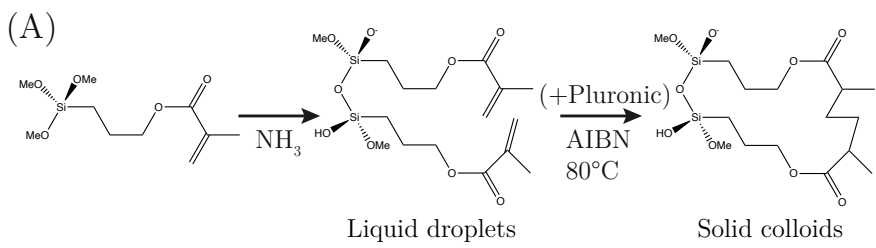

(B)
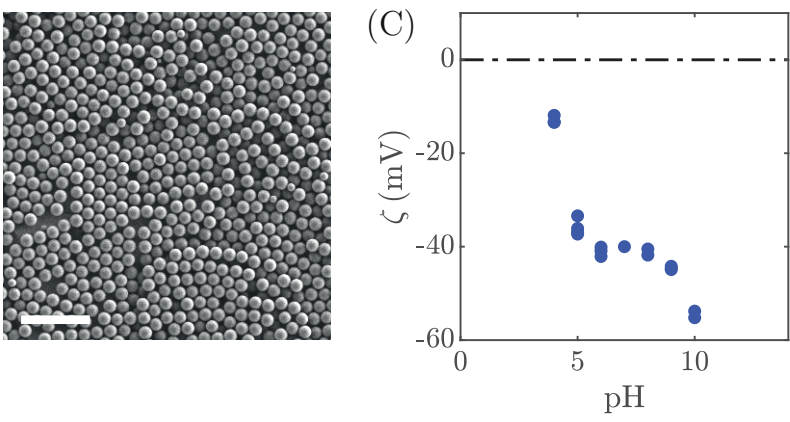

Fig. 2 Synthesis of polymer-coated microspheres. (A) The synthesis of polymeric colloids proceeds in two main steps. First TPM monomers crosslink and nucleate liquid microspheres in basic condition. Pluronic copolymers may be added at this stage and anchor on the colloids. Second, radical polymerization solidifies the colloids and the Pluronic copolymer is permanently grafted on the surface. (B) Monodispersed $1.83 \pm 0.036 \mu \mathrm{m}$-diameter TPM colloids. Scanning electron microscope image. Scale bar: $10 \mu \mathrm{m}$. (C) TPM colloids bear negative surface charges when dispersed in water. The variation of the $\zeta$-potential with $\mathrm{pH}$ reveals that the negative charge becomes stronger as the solution becomes more basic. Background salt concentration: $[\mathrm{NaCl}]=5 \mathrm{mM}$.

\section{Results and discussions}

\subsection{Sweeping the Debye length to investigate polymer-brush properties}

To investigate the properties of the different Pluronic brushes, we measure the electrophoretic mobilities of the polymer-grafted and bare TPM particles by systematically varying the buffer salt concentration within the range $[0.5 \mathrm{mM}-40 \mathrm{mM}]$. In order to vary the interaction between ions and colloids, we perform the exper- iments using different monovalent ( $\mathrm{LiCl}, \mathrm{NaCl}, \mathrm{KCl}$, and $\mathrm{CsCl}$ ) or divalent $\left(\mathrm{MgCl}_{2}, \mathrm{CaCl}_{2}\right)$ salts and different $\mathrm{pH}$ to alter the native surface charge [Fig. 3].

Our data shows a number of important trends [Fig. 3(B-D)]. First, the reduction of the Debye length yields a reduction in particle mobility, a direct consequence of the reduced electric driving force on the Debye layer for a particle of fixed surface charge density $^{39}$. Second, at a fixed Debye length, the addition of a brush reduces the electrophoretic mobility: the longer the polymer, the larger the reduction. Finally, measurements with divalent cations $\left(\mathrm{CaCl}_{2}\right.$ and $\left.\mathrm{MgCl}_{2}\right)$ show reduced electrophoretic mobilities in comparison with monovalent salts at fixed Debye length, indicative of the absorption of the divalent cations on the particles' surface [Fig. 3(B), plain symbols].

Remarkably, for a given polymer brush, the electrophoretic mobility data collapse when normalized by the mobilities of their bare counterparts, $\tilde{\mu}=\mu / \mu_{\text {bare }}$, regardless of the nature of the salt [Fig. 3(C)]. In particular, while the mobilities measured using monovalent and divalent cations differ significantly, indicative of some changes in surface chemistry, their corresponding normalized mobilities collapse. Furthermore, we observe that the normalized mobilities of particles with a short brush, such as Pluronic F68, do not vanish at small $\lambda_{\mathrm{D}}$ [Fig. 3(C)]. This experimental observation invalidates the elemental picture by which the brush adds infinite dissipation on its extension and shifts the no-slip boundary condition by a distance $L$, causing $\tilde{\mu}$ to vanish as $\tilde{\mu}=\exp \left(-L / \lambda_{\mathrm{D}}\right)^{28,30}$.

Next, we modify the surface charge of the colloids by changing the $\mathrm{pH}$ of the solution [Fig. 2(C)] and measure the electrophoretic mobility of particles with different polymer-coating using $\mathrm{NaCl}$ solutions of variable concentration. Despite notable differences in their raw mobilities, indicative of the alteration of the surface chemistry by $\mathrm{pH}$ [Fig. 3(D) inset], the normalized mobility $\tilde{\mu}$ collapse for all tested $\mathrm{pH} \mathrm{4,7}$ and 10 and particles batch [Figure 3(D)]. Those results underline that the normalized mobility $\tilde{\mu}$ is solely specified by the polymer coating, irrespective of properties of the underlying surface: native charge or adsorption of chemical species. This property of the rescaled mobility has not been reported previously and constitutes an important result of the present work. It allows for solely probing the properties of the polymer-brush as the Debye length is varied. Following, the remainder of the manuscript will focus on developing a simple model to rationalize the observed collapse and extract hydrodynamic characteristics of the coated polymer brush from the obtained master-curves.

\subsection{Hydrodynamic model}

We now introduce a minimal model to rationalize the collapse of the normalized mobility $\tilde{\mu}$ into master curves, allowing us to extract the hydrodynamic fingerprint of the polymer coating: the brush thickness and permeability. The collapse of $\tilde{\mu}$ for various surface conditions allows us to focus our discussion on the polymer brush alone. It is worth noting, however, that the brush itself could potentially alter the surface charge, would it be grafted by bonding to surface charge groups. In this case, the reduction of 

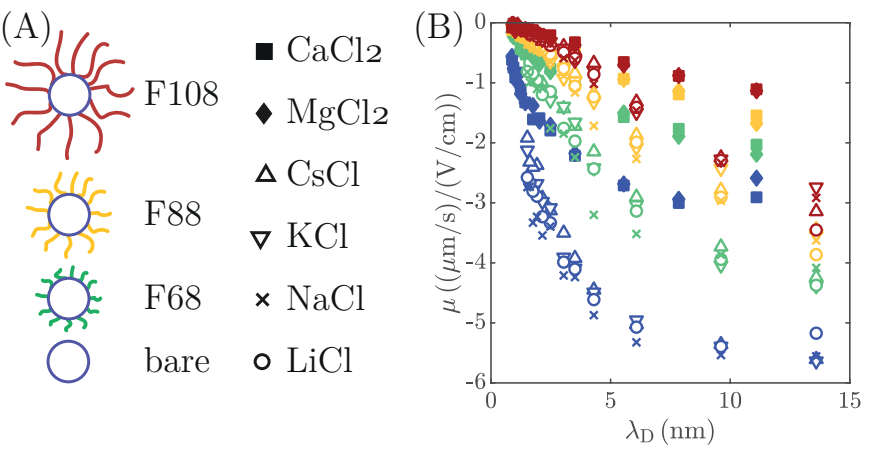
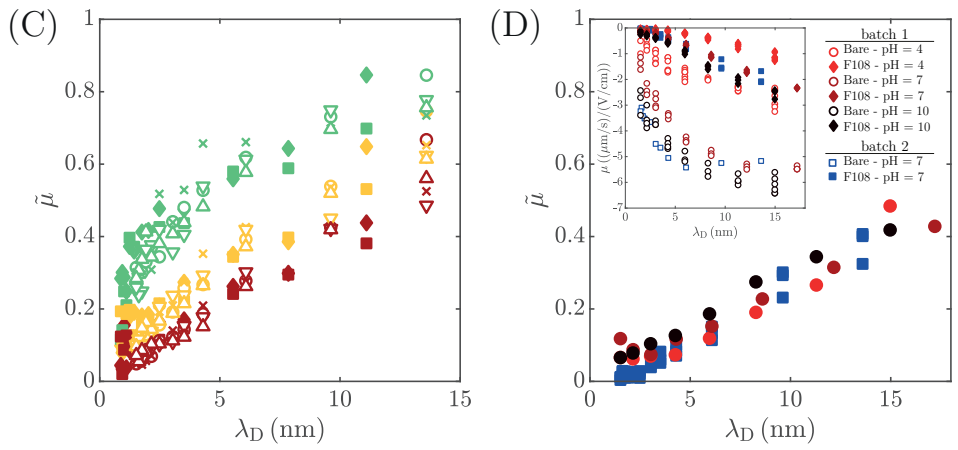

Fig. 3 Evaluating the brush contribution to the change of electrophoretic mobility. (A) Schematics of the bare and polymer-coated TPM particles. The surface of bare TPM particles (blue) is modified to contain polymers of increasing size F68 (green), F88 (yellow) and F108 (red). (B) Electrophoretic mobilities of the bare and polymer-coated particles measured with a zetameter using different salts and various Debye lengths at fixed $\mathrm{pH}=7$. Symbols and colors as set in (A). (C) Normalized mobility $\tilde{\mu}=\mu / \mu_{\text {bare }}$ as a function of the Debye length, showing a collapse of the data of (B) for each polymer brush. The normalized mobility uniquely depends on the polymer brush and is independent of changes in the surface chemistry [see Main Text]. Symbols and colors as set in (A). (D) Inset: mobilities as a function of the Debye length for bare and F108 grafted particles at various pH. The changes in mobilities with $\mathrm{pH}$ reflect changes in surface charges. Blue and red markers stand for two different batches of particles. Main figure: Corresponding normalized mobility as a function of the Debye length, for F108-coated particles and at different $\mathrm{pH}$. The data in acidic $(\mathrm{pH}=4)$, neutral $(\mathrm{pH}=7)$ and basic environments $(\mathrm{pH}=10)$ collapse.

$\tilde{\mu}$ would be independent of the Debye length, in contrast with the strong variations observed in Fig. 3(C). We expect that in the case of neutral polymers such as Pluronic, polymer brushes would not strongly affect the electrostatic properties of the system. We henceforth describe the polymer brush by focusing on its hydrodynamic effects and model the brush as a homogeneous porous medium of finite permeability $\kappa$. We neglect more subtle effects like changes in near-surface permittivity which could be relevant in other settings ${ }^{40}$. We set ourselves in the thin Debye layer approximation, where the radius of the colloidal particles is much larger than the Debye length, in agreement with the experiment [see Fig. 2(B)] and place ourselves in the standard Debye-Hückel approximation. We derive the electro-osmotic flow in this minimal model and show that it captures our experimental observations [Fig. 4(C)].

We consider the simplified 2-dimensional geometry shown in Fig. 1(C). The brush extends to a distance $z=L$ from the surface. The charge density $\rho$ follows the Debye approximation with $\rho(z)=\rho_{0} \exp \left(-z / \lambda_{\mathrm{D}}\right)$, where $\lambda_{\mathrm{D}}$ is the Debye length and $\rho_{0}$ is the charge density at the surface. We consider neutral polymer brushes which do not interact with the cloud of ions and are modeled as a homogeneous and continuous medium, an assumption that would breakdown in situations of sparse coverage. Within this minimal framework, finding the mobility of the particles consists of evaluating the solvent flow $\mathbf{v}$ with respect to the surface in the limit $z \rightarrow \infty$, knowing that $\mathbf{v}_{\mathrm{EP}}=-\mathbf{v}(z \rightarrow \infty)$. This is done by solving the Stokes equation:

$$
\eta \nabla^{2} \mathbf{v}-\alpha_{L}(z) \mathbf{v}+\rho(z) \mathbf{E}_{\mathbf{0}}-\nabla p=0,
$$

where $\eta$ is the viscosity of the solvent, $p$ is the pressure and $\mathbf{E}_{\mathbf{0}}=E_{0} \hat{\mathbf{x}}$ the stationary longitudinal electric field [Fig. 1(C)]. The friction coefficient $\alpha_{L}(z)$ is the step-function given by:

$$
\alpha_{L}(z)= \begin{cases}\eta / \kappa & \text { if } z \leq L \\ 0 & \text { if } z>L\end{cases}
$$

It is worth noting that three length-scales compete in this model: the Debye length $\lambda_{\mathrm{D}}$, the brush length $L$, and the Brinkman length $\xi=\sqrt{\kappa}$, that defines the penetration length of a flow in the porous medium. Their interplay ultimately dictates the profile of the electro-osmotic flow generated from the surface. Unlike the electro-osmosis occurring within charged porous media ${ }^{24}$ the flow only originates from the surface charges at $z=0$ and the role of the brush is purely dissipative, adding drag onto the electroosmotic flow. Remarkably, some sets of $L, \lambda_{\mathrm{D}}$, and $\xi$ yield nonmonotonic flow profiles, as illustrated in Fig. 4(A). The general expression of $\mathbf{v}=v(z) \hat{\mathbf{x}}$, and in the limiting case of an infinite but permeable brush $(L=+\infty, \xi>0)$ are presented in Appendix A.3.

Solving Eq. (1) eventually yields the following expression for the electrophoretic mobility, with $v_{\mathrm{EP}}=\mu E_{0}$ :

$$
\mu=\mu_{\text {bare }} \exp \left(-\frac{L}{\lambda_{\mathrm{D}}}\right) \frac{\left(\frac{\lambda_{\mathrm{D}}}{\xi}\right)^{2}-\exp \left(\frac{L}{\lambda_{\mathrm{D}}}\right) \operatorname{sech}\left(\frac{L}{\xi}\right)+\frac{\lambda_{\mathrm{D}}}{\xi} \tanh \left(\frac{L}{\xi}\right)}{\left(\frac{\lambda_{\mathrm{D}}}{\xi}\right)^{2}-1},
$$

where $\mu_{\text {bare }}=\rho_{0} \lambda_{\mathrm{D}}^{2} / \eta$ is the Smoluchowski mobility of the bare particles. Importantly, Eq. (3) shows that the normalized mobility $\tilde{\mu}$ does not depend on the surface charges, hence supporting the collapse obtained in Fig. 3(B). Figure 4(B) shows the variations of $\tilde{\mu}$ with $\lambda_{\mathrm{D}} / L$ for various values of $\xi / L$. The mobility is reduced as the brush becomes less permeable (i.e. $\xi$ decreases). It eventually follows the simpler trend predicted for a non-permeable brush $\tilde{\mu}=\exp \left(-L / \lambda_{\mathrm{D}}\right)$ [Fig. 4(B), black curve], a situation that has been assumed in previous work ${ }^{30}$. Furthermore, Eq. (3) predicts that $\tilde{\mu}$ monotonously goes from 1 at $\lambda_{\mathrm{D}}=+\infty$ to $\operatorname{sech}(L / \xi)$ as $\lambda_{\mathrm{D}} \rightarrow 0$, [Fig. 4(B), inset].

The non-vanishing electrophoretic mobility as $\lambda_{\mathrm{D}}=0$ originates in the finite permeability of the brush and successfully accounts for the experimental measurements, as shown in Fig. 4C, where fits to the model have been performed for each of the three polymer investigated, regardless of the type of salt. Fitting our experimental data, we estimate $L$ and $\xi$ for different polymer brush. 

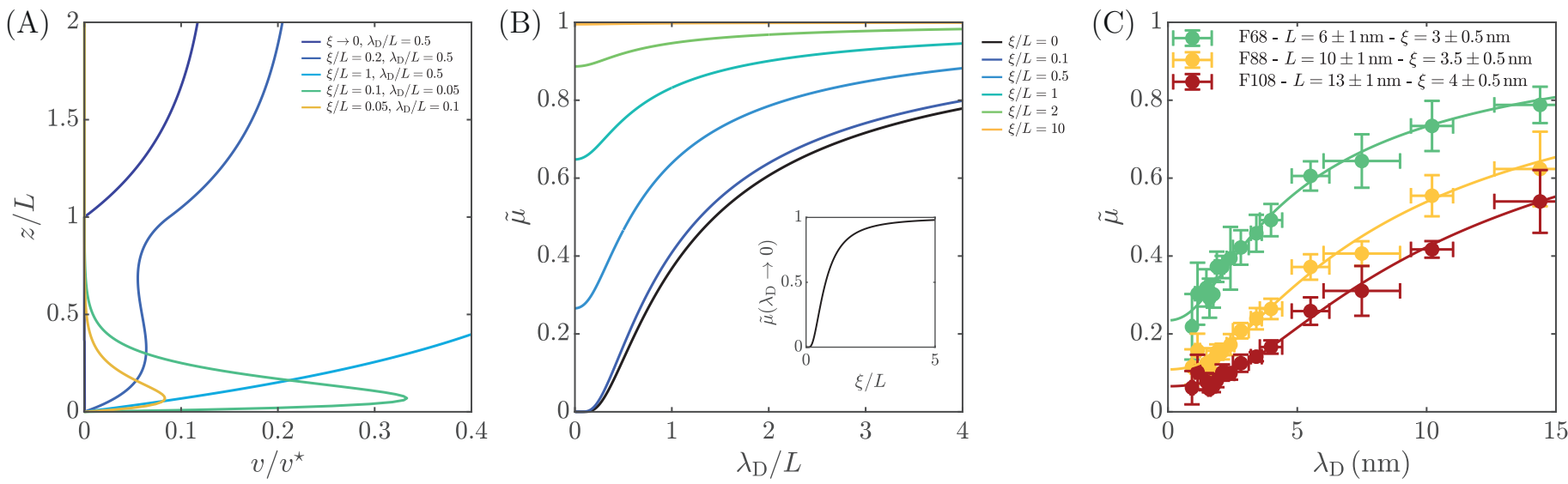

Fig. 4 Electrophoresis of porous polymer brushes. (A) A variety of flow profiles result from the interplay of $L$, $\xi$ and $\lambda_{\mathrm{D}}$. Non-monotonic profiles typically arise when $L$ is large. (B) The normalized mobility decreases as the Debye length decreases. This reduction is weaker for highly permeable brushes (yellow curve) than for non-permeable ones (black curve). Inset: the residual mobility at vanishing Debye length increases with the permeability. (C) The model captures the variations of $\tilde{\mu}$ with $\lambda_{\mathrm{D}}$ for the pluronic brushes investigated in Fig. 3 . It yields $L=6 \pm 1 \mathrm{~nm}$ for F68, $L=10 \pm 1 \mathrm{~nm}$ for F88 and $L=13 \pm 1 \mathrm{~nm}$ for F108. The three pluronic share a roughly common permeability given by $\xi \sim 3 \mathrm{~nm}$.

The values we obtain are reported in Table 1 and compared with independent measurements on nanoparticles (below) and previous results from the literature gathered in Table 2. Remarkably, the measured brush lengths reflect the increasing size of the PEO blocks from F68 to F88 to F108, indicating that the method is sensitive to differences of order $\sim 1 \mathrm{~nm}$.

\subsection{Discussion}

To validate the estimates of brush lengths obtained by electrophoretic measurements, we perform independent measurements of the polymer thickness by estimating hydrodynamic radii of the particles using dynamic light scattering. We synthesize $\sim 200 \mathrm{~nm}$ bare or polymer-coated TPM nanoparticles, in order to get sufficient resolution on the difference of observed hydrodynamic radii [see Appendix A.1, Fig. 6], an unnecessary requirement for measurements done by varying the Debye length and measuring the electrophoretic mobility using a zetameter, as we have previously described. The comparison of the hydrodynamic radii of bare and polymer-coated particles leads to $L_{108}^{\text {hydro }}=19.5 \pm 3 \mathrm{~nm}$ and $L_{88}^{\text {hydro }}=8.6 \pm 4 \mathrm{~nm}$, values comparable to the one reported for Pluronic-coated polystyrene particles ${ }^{41}$.

Unlike dynamic light scattering, we have seen that electrophoretic measurements can also provide an estimate for the brush permeability given by the Brinkman length $\xi$. It is therefore linked to the density of packing of the polymer constituting the brush: the smaller $\xi$ the denser the brush. In order to estimate the polymer volume fraction, we use the model by Hill and coworkers ${ }^{26}$. In this model, the polymer is modeled as a chain of connected segments represented by identical spheres of radius $a$ with a drag coefficient given by Stokes law $C_{\mathrm{drag}}=6 \pi \eta a$. Assuming no interaction between the segments, the friction coefficient is given by $\alpha_{L}=\eta / \xi^{2}=\phi /\left(4 \pi a^{3} / 3\right) C_{\text {drag }}$ where $\phi$ is the brush volume fraction, yielding $\phi=2 / 9(a / \xi)^{2}$. The uncertainty and quadratic dependence on $a$ makes a precise estimate finicky. Using $a=1 \mathrm{~nm}$, we estimate $\phi \sim 1.5 \%$ for F108. This estimate is in line with the literature ${ }^{42}$ : Stenkamp and Berg report surface den- sities of $\sim 1 \mathrm{mg} / \mathrm{m}^{2}$ for F108 on polystyrene, which corresponds to volume fraction of $\sim 1 \%$. Attempts to measure the weight of the polymer brush, by thermogravimetric analysis (TGA) while burning $\sim 2 \mathrm{mg}$ of colloids, failed, as expected as our estimate would result in a relative change of mass of $\sim 1 \mu \mathrm{g}$, below $0.1 \%$ of mass difference we could confidently achieve.

We have shown that the length and permeability of pluronic brushes grafted on TPM particles are accessible through the variation of the normalized mobility $\tilde{\mu}\left(\lambda_{\mathrm{D}}\right)$. Let us detail the range of applicability of this method. A key element is the independence of $\tilde{\mu}\left(\lambda_{\mathrm{D}}\right)$ upon variations of the surface chemistry allowing to isolate, and hence characterize, the influence of the sole brush. This independence holds as long as the brush is neutral. In that case and following a dimensional analysis, the set of equations Eqs. 1 and 2 necessarily gives $v \propto \rho_{0} E_{0} / \eta \times$ length $^{2}$. The electrophoretic velocity can thus be generically written as $v_{\mathrm{EP}}=v_{\text {Smoluchowski }} \times f\left(\lambda_{\mathrm{D}}, L, \xi\right)$ where $f$ is an dimensionless function of $\lambda_{\mathrm{D}}, L$ and $\xi$ that encompasses the properties of the brush, and $v_{\text {Smoluchowski }} \equiv \mu_{\text {bare }} E_{0}$ the solution in the absence of any brush. This condition is satisfied as long as the brush is uncharged, but breaks down in the case of polyelectrolyte brushes. Importantly, this scaling is robust to the specifics of the brush conformation. It makes the normalized mobility $\tilde{\mu}$ the right observable to investigate neutral polymer coatings without a priori knowledge of their conformation as we illustrate in the following section.

\subsection{Probing nanoscale features of adsorbed polymer brushes}

We now apply our approach to gain insight into polymer adsorption. We perform Debye length sweeps and compare the normalized electrophoretic mobilities of particles with adsorbed neutral polymers having different affinities with the surface. We use Pluronic F108 and poly(ethylene oxide) (PEO) polymers with comparable sizes and perform electrophoretic mobility measure- 
ments at different polymer concentrations.

We consider TPM particles, which are initially bare, and let either Pluronic F108 or PEO adsorb onto their surface. We use PEO with molecular mass of $35 \times 10^{3} \mathrm{~g} / \mathrm{mol}$ for which twice the radius of gyration $2 R_{\mathrm{g}} \sim 11 \mathrm{~nm}$ is comparable to the length of the F108 brush. The evolution of $\tilde{\mu}$ for varying Debye lengths is shown in Fig. 5(A) for F108 and in Fig. 5(B) for PEO. In both cases, the normalized electrophoretic mobilities $\tilde{\mu}$ systematically decrease upon increasing the polymer concentration, as more polymer becomes adsorbed onto the particle surface. They eventually saturate for $[\mathrm{F} 108] \geq 10^{-2} \mathrm{~mol} / \mathrm{L}$ and $[\mathrm{PEO}] \geq 10^{-5} \mathrm{~mol} / \mathrm{L}$, which indicates the formation of a maximally packed polymer brush on the surface of the TPM particles.

(A)

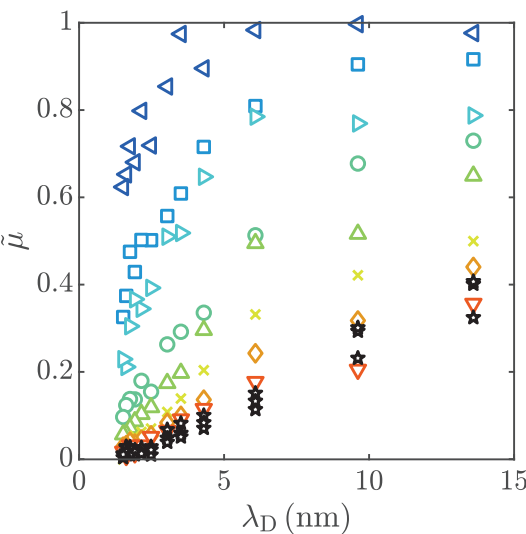

(B)

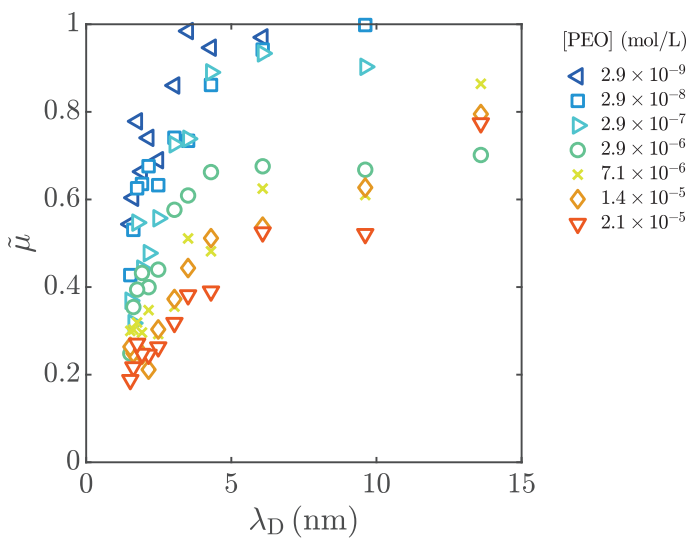

Fig. 5 Polymer adsorption probed by Debye length sweeps. (A) Reduction of the normalized mobility with $\lambda_{\mathrm{D}}$ for TPM colloidal particles with adsorbed F108. (B) Reduction of the normalized mobility with $\lambda_{\mathrm{D}}$ for the same TPM colloidal particles with adsorbed PEO.

By comparing in Fig. 5(A) the normalized mobilities for adsorbed F108 and grafted F108 at saturation, we find the two indistinguishable. This remarkable collapse shows that the polymer coating exhibits identical hydrodynamic behavior after adsorption or grafting protocols (see Section 2, Appendix A.1 and Fig. 7), and suggests similar conformations at saturation. Such measurement is in contrast with existing views that the hydrophobic poly(propylene oxide) (PPO) part of the triblock copolymer would anchor more strongly to a liquid particle than on a solid one $^{15}$.
Comparing the sets of curves for F108 and PEO points towards differences in the adsorption of the two polymers on TPM particles. In particular, adsorbed PEO leads to a much weaker reduction of electrophoretic mobility at saturation than adsorbed F108, suggesting that the PEO coating is more porous than the F108 brush. This is consistent with the hydrophilic nature of PEO and the absence of hydrophobic moiety, which lead to a weaker adsorption than the triblock PEO-PPO-PEO. More quantitatively, Eq. 3 fails to capture the evolution of $\tilde{\mu}$ for PEO coatings, presumably because such sparse coatings strongly escape the description as a continuous porous medium of homogeneous permeability (Eq. 2). Conversely, the good agreement of Fig. 4(C) between theory and experiments on Pluronic brushes validates this simple description. It suggests that the Pluronic brushes are in extended conformations, well described by step profiles as put forward by Alexander and de Gennes ${ }^{37,43}$. Achieving a similar agreement between theory and experiments for sparser coatings, such as PEO adsorbed on TPM, would require a more faithful modeling of the polymer conformation via $\alpha_{L}(z)$. In turn, further theoretical developments relative to the conformation of neutral polymer coating will benefit from the quantitative insights and constraints provided by our approach.

\section{Conclusion}

We have measured the electrophoretic mobility of polymercoated particles at various Debye lengths in different electrolytes and at different $\mathrm{pH}$. We have evidenced that changes in surface chemistry can be disregarded by normalizing the mobility of polymer-coated particles with the mobility of bare particles. The variations of such normalized mobilities with the Debye length depend on the characteristics of the polymer brush alone. Our measurements are accounted for by a purely hydrodynamic model which describes the brush as a neutral and homogeneous porous medium with finite permeability. Our approach is directly applicable to other settings and we show that it captures differences in coatings of PEO-PPO-PEO block copolymer and PEO polymer.

Conceptually, this work shows that the Debye length can be used as a faithful probe that offers perspectives on the adsorption of polymers onto colloidal particles. In particular, the measure of normalized mobility $\tilde{\mu}\left(\lambda_{\mathrm{D}}\right)$ will provide a robust test for the development of theoretical models. Practically, this work provides a valuable tool for the rapid and convenient characterization of neutral polymer brushes on particle surfaces using conventional zetameters. Their widespread use in physico-chemistry laboratories together with the increasingly broad applications of polymer brushes in colloidal science suggest a large prospect to our method.

\section{A Appendices}

\section{A.1 Materials and Methods Preparation of bare TPM Particles}

TPM particles are typically prepared by adding $1 \mathrm{~mL}$ of 3 (Trimethoxysilyl)propyl methacrylate ( $\geq 98 \%$, purchased from Sigma-Aldrich) to a solution of $200 \mathrm{~mL}$ deionized water contain- 
ing $200 \mu \mathrm{L}$ of $28 \% \mathrm{NH}_{3}$ under stirring. After 3 hours, the TPM particles are solidified via a radical polymerization to obtain bare TPM particles. The suspension is first diluted by a factor of two in deionized water, and the polymerization is achieved by adding $\sim 1 \mathrm{mg}$ of 2,2'-Azobis(2-methylpropionitrile) (AIBN, $\geq 98 \%$ purchased from Sigma-Aldrich) and heating the mixture at $80^{\circ} \mathrm{C}$ for 3 hours. TPM particles are purified via repeated cycles of sedimentation and resuspension in deionized water. The above procedure yields monodisperse particles of $1.83 \pm 0.036 \mu \mathrm{m}$-diameter [Fig. 2(B)]. Variations in the amounts of ammonia and TPM monomer added enable the synthesis of particles with a wide range of sizes ${ }^{36}$. We use $\sim 200 \mathrm{~nm}$-diameter particles for dynamic light scattering measurements of the brush thickness.

\section{Grafting polymer brushes onto TPM particles}

To graft a polymer brushes onto the surface of TPM particles, polymers are introduced to an emulsion containing liquid TPM particles in water. To functionalize the TPM oil droplets with copolymers of 3 different lengths, Pluronic F68, F88, and F108 are used. The copolymers are added to the emulsion to a final concentration of $0.05 \mathrm{wt} \%$ under stirring before polymerization. The particles are then polymerized and purified as described above for bare TPM particles.

\section{Adsorbing polymer brushes onto TPM particles}

To adsorb polymers onto TPM particles, polymer solutions are simply introduced to a suspension of bare particles under stirring. Samples are left to homogenize for a few hours before the mobilities are measured. We verify that the particles have reached equilibrium adsorption by comparing the electrophoretic measurements with measurements performed at a later time. The saturation in mobility of particles adsorbed with F108 brushes is notably reached for a concentration comparable with the critical micellar concentration $[\mathrm{F} 108] \sim 3.5 \mathrm{mM}$ at room temperature ${ }^{44}$. Comparison with saturated grafted brushes is made in Fig. 5(A) with copolymer added to the emulsion to final concentrations $1 \mathrm{wt} \%, 2 \mathrm{wt} \%$ and $4 \mathrm{wt} \%$.

\section{Instrumentation}

All mobility measurements were taken using a Malvern Zetasizer (nano series).

\section{A.2 Characterization of Pluronic-coated TPM colloids}

Table 1 Properties of Pluronic brushes on TPM measured in this Article.

\begin{tabular}{llll}
\hline Polymer & $\xi /$ TPM & $L /$ TPM & $L^{\text {hydro }} /$ TPM \\
\hline F68 & $3 \pm 0.5 \mathrm{~nm}$ & $6 \pm 1 \mathrm{~nm}$ & \\
F88 & $3.5 \pm 0.5 \mathrm{~nm}$ & $10 \pm 1 \mathrm{~nm}$ & $8.5 \pm 4 \mathrm{~nm}$ \\
F108 & $4 \pm 0.5 \mathrm{~nm}$ & $13 \pm 1 \mathrm{~nm}$ & $20 \pm 3 \mathrm{~nm}$ \\
\hline
\end{tabular}

Table 2 Length of Pluronic brushes on polystyrene (PS) colloids from the literature.

\begin{tabular}{llll}
\hline Polymer & $L /$ PS $^{45}$ & $L^{\text {hydro }} /$ PS $^{41}$ & $L^{\text {steric }} /$ PS $^{46}$ \\
\hline F68 & & $5.7 \mathrm{~nm}$ & \\
F88 & $8.0 \mathrm{~nm}$ & $8.0 \mathrm{~nm}$ & \\
F108 & $10.2 \mathrm{~nm}$ & $10.6 \mathrm{~nm}$ & $18.5 \mathrm{~nm}$ \\
\hline
\end{tabular}
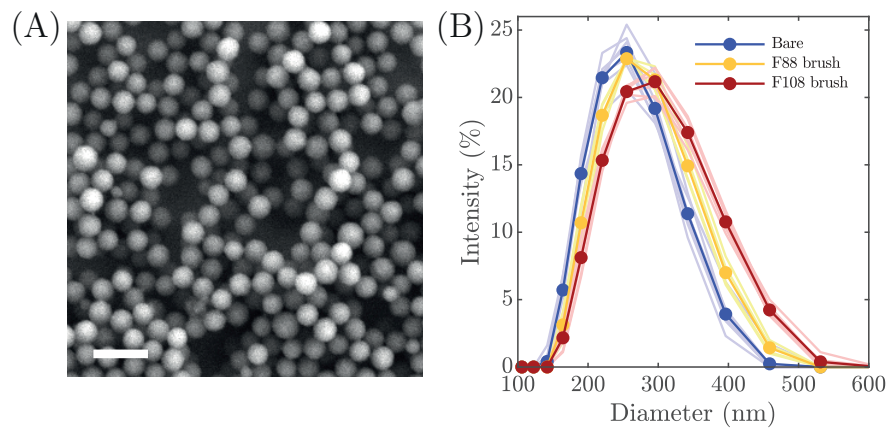

Fig. 6 Dynamic light scattering estimates of the length of polymer brushes. (A) Small $200 \mathrm{~nm}$-diameter TPM colloids observed by scanning electron microscopy. Scale bar: $500 \mathrm{~nm}$. (B) The size distributions obtained via dynamic light scattering indicate that polymer brushes extend about $19.5 \mathrm{~nm}$ (F108) and $8.6 \mathrm{~nm}$ (F88). Independant measurements (pale curves) were performed and each was fitted by a log-normal distribution to give an estimate of $L^{\text {hydro }}$. Their average and standard deviation are reported in Table 1. Solid curves: averages of the pale curves.

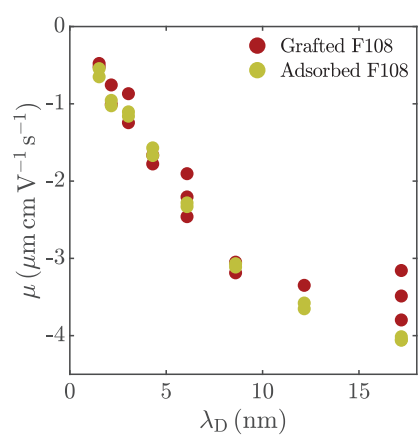

Fig. 7 Electrophoretic mobilities of grafted and adsorbed F108 brushes. Grafted and adsorbed F108 brushes show similar variations of the electrophoretic mobility of particles $\mu$ with the Debye length $\lambda_{\mathrm{D}}$. Red markers: TPM particles with F108 grafted on the surface before polymerization. Yellow markers: TPM particles with saturated adsorbed F108, washed by several cycles of centrifugation and resuspension in de-ionized water.

\section{A.3 Flow profiles within the brush and beyond}

Solving Stokes equation [Eq. (1)] with the brush being described by Eq. (2) yields the flow profile $\mathbf{v}=v(z) \hat{\mathbf{x}}$ :

$$
\begin{aligned}
v(z)= & \frac{v^{\star}}{\left(\frac{\lambda_{\mathrm{D}}}{\xi}\right)^{2}-1}\left\{\exp \left(-z / \lambda_{\mathrm{D}}\right)-\cosh (z / \xi)+\sinh (z / \xi)\right. \\
& {\left.\left[\exp \left(-L / \lambda_{\mathrm{D}}\right) \frac{\lambda_{\mathrm{D}}}{\xi} \operatorname{sech}(L / \xi)+\tanh (L / \xi)\right]\right\}, }
\end{aligned}
$$

for $z<L$, and:

$$
v(z)=v(L)+v^{\star} \exp \left(-L / \lambda_{\mathrm{D}}\right)\left(1-\exp \left(-\frac{z-L}{\lambda_{\mathrm{D}}}\right)\right),
$$

for $z>L$, where $v(L)$ is evaluated using Eq. (4) and $v^{\star}=\mu_{\mathrm{bare}} E_{0}$ is the Smoluchovski velocity.

The interplay of $L, \xi$ and $\lambda_{\mathrm{D}}$ gives rise to a variety of velocity profiles, as shown in Fig. 4A. It includes non-monotonic profiles 
which arise when $L$ is large with respect to $\xi$ and $\lambda_{\mathrm{D}}$. In particular in the limit of an infinite brush for which the velocity profile reduces to:

$$
v(z)=\frac{v^{\star}}{\left(\frac{\lambda_{\mathrm{D}}}{\xi}\right)^{2}-1}\left[\exp \left(-z / \lambda_{\mathrm{D}}\right)-\cosh (z / \xi)\right]
$$

As the polymer brush is uncharged, the force that drives the flow solely originates from the Debye layer, while damping occurs within the whole brush. Consequently, such coating enables the localization of electro-osmotic flow at the surface of a charged substrate unlike the typical electro-osmotic plug flows.

\section{References}

1 R. J. Hunter, Foundations of colloid science, Oxford University Press, USA, 1989.

2 S. Sacanna and D. J. Pine, Current Opinion in Colloid \& Interface Science, 2011, 16, 96-105.

3 S. Sacanna, W. T. M. Irvine, P. M. Chaikin and D. J. Pine, Nature, 2010, 464, 575-578.

4 E. Duguet, A. Désert, A. Perro and S. Ravaine, Chem. Soc. Rev., 2011, 40, 941-21.

5 Y. Wang, Y. Wang, X. Zheng, É. Ducrot, J. S. Yodh, M. Weck and D. J. Pine, Nature Communications, 2015, 6, 1-8.

6 Y. Wang, Y. Wang, D. R. Breed, V. N. Manoharan, L. Feng, A. D. Hollingsworth, M. Weck and D. J. Pine, Nature, 2012, 490, 51-55.

7 W. B. Rogers and J. C. Crocker, Proceedings Of The National Academy Of Sciences of The United States Of America, 2011, 108, 15687-15692.

8 M. P. Valignat, O. Theodoly, J. C. Crocker, W. B. Russel and P. M. Chaikin, Proceedings Of The National Academy Of Sciences Of The United States Of America, 2005, 102, 4225-4229.

9 J.-G. Park, S.-H. Kim, S. Magkiriadou, T. M. Choi, Y.-S. Kim and V. N. Manoharan, Angewandte Chemie-International Edition In English, 2014, 53, 2899-2903.

10 É. Ducrot, M. He, G.-R. Yi and D. J. Pine, Nature Materials, 2017, 16, 652-657.

11 P. G. de Gennes, Adv. Colloid Interface Sci., 1987, 27, 189209.

12 S. T. Milner, T. A. Witten and M. E. Cates, Macromolecules, 1988, 21, 2610-2619.

13 S. T. Milner, Science, 1991, 251, 905-914.

14 A. M. Bodratti, B. Sarkar and P. Alexandridis, Advances in Colloid and Interface Science, 2017, 244, 132-163.

15 J. A. Shar, T. M. Obey and T. Cosgrove, Colloids And Surfaces A-Physicochemical And Engineering Aspects, 1998, 136, 21-33.

16 J. L. Anderson, Annual Review Of Fluid Mechanics, 1989, 21, 61-99.

17 L. Bocquet and E. Charlaix, Chem. Soc. Rev., 2010, 39, 10731095.

18 A. Ajdari and L. Bocquet, Physical Review Letters, 2006, 96, 186102.

19 T. M. Squires, Physics of Fluids, 2008, 20, 092105.

20 R. J. Messinger and T. M. Squires, Physical Review Letters,
2010, 105, 144503.

21 P. Wiersema, A. Loeb and J. T. G. Overbeek, Journal of Colloid and Interface Science, 1966, 22, 78-99.

22 G. J. Fleer, Polymer adsorption and its effect on colloidal stability, Veenman, 1971.

23 L. Koopal, V. Hlady and J. Lyklema, Journal of colloid and interface science, 1988, 121, 49-62.

24 J. Irigoyen, V. B. Arekalyan, Z. Navoyan, J. Iturri, S. E. Moya and E. Donath, Soft Matter, 2013, 9, 11609-9.

25 J. A. Cohen and V. A. Khorosheva, Colloids and Surfaces A: Physicochemical and Engineering Aspects, 2001, 195, 113-127.

26 R. J. Hill, D. A. Saville and W. B. Russel, Journal of Colloid And Interface Science, 2003, 258, 56-74.

27 R. Qiao, Langmuir, 2006, 22, 7096-7100.

28 G. Paumier, J. Sudor, A.-M. Gue, F. Vinet, M. Li, Y. J. Chabal, A. Estève and M. Djafari-Rouhani, Electrophoresis, 2008, 29, 1245-1252.

29 T. López-León, J. L. Ortega-Vinuesa, D. Bastos-González and A. Elaïssari, J. Phys. Chem. B, 2006, 110, 4629-4636.

30 T. J. Barnes and C. A. Prestidge, Langmuir, 2000, 16, 41164121.

31 W. Hellmich, J. Regtmeier, T. T. Duong, R. Ros, D. Anselmetti and A. Ros, Langmuir, 2005, 21, 7551-7557.

32 S. Ahualli, M. Ballauff, F. J. Arroyo, A. V. Delgado and M. L. Jiménez, Langmuir, 2012, 28, 16372-16381.

33 J. Janzen, X. Song and D. Brooks, Biophysical Journal, 1996, 70, $313-320$.

34 K. Devanand and J. Selser, Macromolecules, 1991, 24, 59435947.

35 S. Bekiranov, R. Bruinsma and P. Pincus, Physical Review E, 1997, 55, 577.

36 C. Van Der Wel, R. K. Bhan, R. W. Verweij, H. C. Frijters, Z. Gong, A. D. Hollingsworth, S. Sacanna and D. J. Kraft, Langmuir, 2017, 33, 8174-8180.

37 M. Rubinstein, R. H. Colby et al., Polymer physics, Oxford university press New York, 2003, vol. 23, pp. 186-187.

38 S. T. Milner, T. Witten and M. Cates, EPL (Europhysics Letters), 1988, 5, 413.

39 K. Makino and H. Ohshima, Langmuir, 2010, 26, 18016 18019.

40 D. J. Bonthuis and R. R. Netz, The Journal of Physical Chemistry $B, 2013,117,11397-11413$.

41 J. Shar, T. Obey and T. Cosgrove, Colloids and Surfaces A: Physicochemical and Engineering Aspects, 1998, 136, 21-33.

42 V. S. Stenkamp and J. C. Berg, Langmuir, 1997, 13, 38273832.

43 S. Alexander, Journal De Physique, 1977, 38, 983-987.

44 P. Alexandridis, J. F. Holzwarth and T. A. Hatton, Macromolecules, 1994, 27, 2414-2425.

45 T. J. Barnes and C. A. Prestidge, Langmuir, 2000, 16, 41164121.

46 M. A. Bevan and D. C. Prieve, Langmuir, 2000, 16, 9274 9281. 